\title{
Glycopeptide resistance in gram-positive bacteria: from black and white to shades of grey
}

\section{Introduction}

The glycopeptide antibiotics vancomycin and teicoplanin are used extensively for the treatment and prophylaxis of infections caused by multi-resistant gram-positive bacteria. Vancomycin was licensed for use in 1958, although it was not used extensively until the 1980s. During the intervening period there was little evidence of the emergence of bacterial resistance. However, since the mid-1980s glycopeptide resistance has been detected increasingly among clinical isolates of gram-positive bacteria in many countries. ${ }^{1} \mathrm{Re}$ sistance to glycopeptides appears to be an intrinsic characteristic of most members of the genera Leuconostoc, Pediococcus and Nocardia, of some species of Lactobacillus and of Erysipelothrix rhusiopathiae. However, these organisms are isolated only occasionally from clinical specimens. Resistance is encountered most often in enterococci, and has also been described in Staphylococcus spp. Glycopeptide resistance in staphylococci has been found mainly in coagulasenegative species, although the emergence of clinical isolates of glycopeptide-resistant $S$. aureus remains a concern. Glycopeptide-resistant organisms show considerable phenotypic and genotypic heterogeneity even within the confines of a single genus or species. The importance of detecting and identifying glycopeptideresistant organisms correctly in the clinical laboratory has been discussed elsewhere. ${ }^{2}$ Here, we provide an overview of glycopeptide resistance in gram-positive bacteria and discuss the biochemical basis of resistance.

\section{Glycopeptide resistance in enterococci}

The first reports of glycopeptide resistance in the genus Enterococcus described transferable, high-level resistance to vancomycin (MIC $\geqslant 512 \mathrm{mg} / \mathrm{L}$ ) and cross-resistance to teicoplanin (MIC $\geqslant 8 \mathrm{mg} / \mathrm{L}$ ). However, enterococci were soon isolated that did not fit this pattern of resistance; they exhibited nontransferable resistance to lower concentrations of vancomycin (typical MICs up to $128 \mathrm{mg} / \mathrm{L}$ ) and remained sensitive to teicoplanin (MICs $\leqslant 4 \mathrm{mg} / \mathrm{L}$ ). Both forms of resistance were usually inducible (i.e., expressed only after exposure of the organisms to vancomycin) and were associated with the production of novel cytoplasmic membrane proteins. Constitutive low-level resistance to vancomycin, but sensitivity to teicoplanin, was recognised as an intrinsic property of the species $E$. gallinarum and $E$. casseliflavus. $^{3}$ These three glycopeptide resistance phenotypes have been designated VanA, VanB and VanC, respectively.

The genotypic and biochemical basis of each phenotype is now being investigated. Three genes-van $A$, van $B$ and van $C$, which correspond to these phenotypes - have been cloned and sequenced. ${ }^{4-6}$ The van $A$ and $\operatorname{van} B$ genes have each been found by use of DNA probes in vancomycin-resistant clinical isolates of $E$. faecium and $E$. faecalis; van $A$ has also been identified in occasional isolates of vancomycinresistant $E$. avium and $E$. durans. The van $C$ gene appears to be present only in E. gallinarum. Details of the gene(s) responsible for the VanC-like resistance phenotype of $E$. casseliflavus have not been published.

Currently available data indicate that the three phenotypes arise from a common resistance mechanism. The target site for glycopeptide antibiotics is the D-alanyl-D-alanine group at the end of the pentapeptide side-chains of peptidoglycan precursors. This dipeptide is produced by the enzyme D-ala-D-ala ligase. Despite differences in the DNA sequences of the van $A$, van $B$ and van $C$ genes, the three gene products show amino-acid homology with each other and also with the D-ala-D-ala ligase of Escherichia coli. ${ }^{4-6}$ These gene products are ligases with altered substrate specificities and result in the production of altered peptidoglycan side-chains that do not bind glycopeptides and hence confer resistance.

Enterococci of the VanA phenotype produce peptidoglycan precursors with side-chains terminating in D-alanyl-D-lactate. ${ }^{7}$ The $\operatorname{van} A$ gene product forms this depsipeptide, but two other genes (vanH and $\operatorname{van} X$ ) are essential for expression of resistance. The van $H$ gene product supplies D-lactate from pyruvate, but the function of the $\operatorname{van} X$ gene product has not been determined. In the VanA $E$. faecium strain studied in greatest detail, these three genes are located on a transposable element, $\operatorname{Tn} 1546$, of $c .10 \cdot 8 \mathrm{~kb}{ }^{8}$ This transposon also carries two genes (vanS and van $R$ ) that regulate the expression of glycopeptide resistance and two genes (van $Y$ and van $Z$ ) which are not essential for expression of resistance. Other enterococci of the VanA class have been shown to carry similar transposons. The observation that some enterococci of the VanA phenotype are able to transfer glycopeptide resistance in the absence of plasmid DNA led to the suggestion that a conjugative transposon might be involved. ${ }^{9}$ Despite this, no conjugative properties have been associated with $\operatorname{Tn} 1546$. Furthermore, this 
transposon is considerably smaller than most other conjugative transposons characterised previously in enterococci or other genera. The mechanism of transfer of glycopeptide resistance in the absence of plasmid DNA remains unclear.

Although the mechanism of glycopeptide resistance has been determined in more detail for enterococci of the VanA resistance phenotype, the van $B$ and van $C$ genes are also known to encode alternative D-ala-D-ala ligases. The component of the peptidoglycan precursors in enterococci of these phenotypes has not been determined. By analogy with the genetics of the VanA phenotype, it is likely that several genes are necessary for expression of glycopeptide resistance, at least for the VanB phenotype, but this remains to be confirmed.

As more glycopeptide-resistant enterococci have been isolated worldwide, it has become apparent that the form of resistance shown by a strain cannot be inferred reliably from its phenotype alone. Increasingly, enterococci are being isolated that do not fit readily into the recognised resistance phenotypes. In the past, enterococci resistant to teicoplanin as well as vancomycin were all of the VanA class. However, some teicoplanin-resistant clinical isolates have been shown to be constitutive-expression mutants of VanB strains. Conversely, strains have also been identified which hybridise with vanA gene probes, but which are sensitive to teicoplanin. The VanA phenotype is no longer the only transferable form of glycopeptide resistance. Recently, enterococci with transferable Van $B$ resistance have been described, and in some cases the $\operatorname{van} B$ gene was located on plasmid DNA. Furthermore, the $\operatorname{van} B$ gene has been found not only in enterococci with the low-level vancomycin resistance (typical MICs $32-128 \mathrm{mg} / \mathrm{L}$ ) originally associated with this phenotype, but also in strains with highlevel vancomycin resistance (MICs up to $1024 \mathrm{mg} / \mathrm{L}$ ). Clearly, the working definitions of the glycopeptide resistance classes found in enterococci are becoming blurred. In the future, various genotypic methods will have to be used to characterise the type of glycopeptide resistance displayed by clinical isolates, with less emphasis placed on resistance phenotypes.

\section{Vancomycin-dependent enterococci}

Recently, the diagnosis of enterococcal infection has been complicated by the emergence of enterococci dependent on vancomycin. These strains will grow only around vancomycin disks or on media supplemented with vancomycin and, therefore, may not be cultured successfully in many laboratories. In the UK, vancomycin-dependent enterococci have been isolated only from faeces, but they have caused bacteraemia and urinary tract infection in the USA. ${ }^{10,11}$

Vancomycin-dependent enterococci are derived from resistant strains and they have been isolated from patients receiving courses of vancomycin. They appear to have defective D-ala-D-ala ligases as they will grow also on media supplemented with this dipeptide. Under most conditions, such mutations would almost certainly be lethal. However, the selective pressure exerted during vancomycin therapy allows induction of the alternative, "vancomycin-resistant" ligase (i.e., the van $A$ or $\operatorname{van} B$ gene product) and this permits survival. Interestingly, the emergence of these dependent organisms has allowed the concentration of vancomycin needed to induce production of the alterative ligases to be estimated, and for strains tested, vancomycin must be present at concentrations of $0 \cdot 25-2 \mathrm{mg} / \mathrm{L}$ before growth can occur. The extent to which vancomycindependent enterococci will cause clinical problems in the future is unknown, but they will undoubtedly represent a considerable diagnostic challenge.

\section{Glycopeptide resistance in staphylococci}

As with enterococci, glycopeptide-resistance in coagulase-negative staphylococci, and in particular $S$. haemolyticus and $S$. epidermidis, is phenotypically diverse. Some isolates are resistant to both vancomycin and teicoplanin, but the phenotype most frequently reported in staphylococci is teicoplanin resistance (typical MICs $\leqslant 128 \mathrm{mg} / \mathrm{L}$ ) and vancomycin sensitivity (MIC $\leqslant 4 \mathrm{mg} / \mathrm{L}$ ). This phenotype has not been reported in glycopeptide-resistant organisms of other genera. The only report of glycopeptide resistance in a clinical isolate of $S$. aureus is of teicoplanin resistance (MIC $8 \mathrm{mg} / \mathrm{L}$ ) alone. In the latter case, this resistance emerged during teicoplanin therapy of a patient with endocarditis. ${ }^{12}$

In most staphylococci, glycopeptide resistance appears to be expressed constitutively, is not plasmidmediated and is stable in the absence of selective pressure. Several studies have indicated that glycopeptide-resistant staphylococci may have altered cell walls, on the basis of altered colonial morphology, altered susceptibility to lysozyme or lysostaphin and loss of phage and capsular typing characteristics in resistant variants. ${ }^{13,14}$ More direct evidence has come from electronmicroscopy studies in which resistant staphylococci were shown to have thicker, more irregular cell walls than sensitive organisms. ${ }^{13-15}$ However, none of these changes has been seen consistently by all workers and for all strains examined.

In one study, teicoplanin-resistant mutants were derived from a susceptible clinical isolate of $S$. aureus (which subsequently became resistant during teicoplanin therapy of endocarditis) at frequencies that indicated that a single gene was responsible for the glycopeptide resistance. ${ }^{12}$ Further work on this strain indicated that the mutation may lie in a gene involved in the regulation of both penicillin-binding protein 2 and a novel $35-\mathrm{kDa}$ cytoplasmic membrane protein. ${ }^{16}$ Several previous studies have also demonstrated novel membrane proteins (35 and $39 \mathrm{kDa}$ ) in resistant clinical isolates and laboratory mutants of both 
coagulase-negative staphylococci and $S$. aureus, but the role of these proteins in glycopeptide resistance has not been determined. The proteins are similar in size to the glycopeptide-inducible cytoplasmic membrane proteins of VanA and VanB enterococci. However, as these resistant staphylococci produced peptidoglycan precursors which terminate in D-ala-D-ala,${ }^{17}$ these proteins are unlikely to be ligases of altered specificity. Furthermore, Daum and colleagues found no aminoacid homology between a $39-\mathrm{kDa}$ protein in laboratory-derived, glycopeptide-resistant $S$. aureus and the $39-\mathrm{kDa}$ VanA protein from enterococci. ${ }^{14}$

Some resistant isolates of $S$. epidermidis have been shown to have an increased ability to sequester glycopeptides, particularly teicoplanin. ${ }^{18}$ However, other studies have found no differences between the number of glycopeptide molecules bound by sensitive or resistant cells of S. epidermidis or S. haemolyticus. ${ }^{17}$ This may be due partly to differences in the growth phase of the organisms studied. Increased binding of glycopeptides to altered cell walls at sites other than the D-ala-D-ala target site might account for resistance in staphylococci, but this remains to be proven. However, as noted by O'Hare and Reynolds, ${ }^{17}$ any protection of the target sites from the action of glycopeptides would still have to permit normal cell wall biosynthetic enzymes to function unhindered.

Noble and colleagues reported conjugal transfer in vitro of the genes responsible for the enterococcal VanA phenotype from $E$. faecalis to $S$. aureus. ${ }^{19}$ The transconjugants appeared to express inducible resistance to high levels of vancomycin (MICs $\leqslant 1000 \mathrm{mg} / \mathrm{L}$ ), although they could not transfer resistance to other $S$. aureus strains and the resistance was lost in the absence of selective pressure. This demonstration of transfer of the VanA phenotype to $S$. aureus causes particular concern. There is DNA homology between the genes that encode $\beta$-lactamase production and high-level gentamicin resistance in enterococci and staphylococci, ${ }^{3}$ providing precedent for the exchange of resistance genes between these genera. The possible emergence of clinical isolates of $S$. aureus that harbour enterococcal glycopeptide resistance genes has serious implications.

\section{Glycopeptide resistance in other gram-positive genera}

Few studies have addressed the mechanism of glycopeptide resistance in the intrinsically resistant genera Lactobacillus, Leuconostoc, Pediococcus, Erysipelothrix and Nocardia. This probably reflects the lesser clinical importance associated with glycopeptide resistance in these organisms. These genera are usually susceptible to various commonly used antimicrobial agents and glycopeptides would not normally be considered appropriate therapy for infections caused by them. Where resistance mechanisms have been investigated, these intrinsically resistant organisms express constitutive, non-transferable resistance to both vancomycin and teicoplanin.

Although the basis of glycopeptide resistance in these organisms remains to be determined, the mechanism(s) appears to differ from that in enterococci. These intrinsically resistant genera have peptidoglycan precursors which terminate in the usual D-ala-D-ala group and no glycopeptide-inducible proteins have been observed in most isolates studied. Probes specific for enterococcal glycopeptide resistance genes do not hybridise with their DNA. Current data suggest that glycopeptides bind poorly to their cell wall materials and, in contrast with vancomycininduced enterococci of the VanA and VanB classes, the amount of binding is not increased after removal of membrane-associated proteins. ${ }^{20}$ Furthermore, cell membranes from Leu. mesenteroides or P. pentosaceus do not prevent binding of vancomycin to exogenous pentapeptides with terminal D-ala-D-ala groups. These data suggest that membrane-associated proteins do not have a major role in protecting these organisms from glycopeptides. The size of the side-chains of the amino acids present in the pentapeptides of peptidoglycan precursors can affect the affinity of glycopeptide binding. This may account for the intrinsic resistance of these genera, ${ }^{20}$ but further investigation is needed.

An isolate of Lac. viridescens has been described in which glycopeptide resistance appeared to correlate with carriage of plasmid DNA and the appearance of vancomycin-inducible proteins. ${ }^{20}$ Also, amino-acid homology has been described between the vanH geneproduct of VanA class enterococci and the D-lactate dehydrogenases of Lactobacillus and Leuconostoc strains. ${ }^{21}$ Such observations warrant further investigation as they might provide insight into the origin and evolution of the vancomycin resistance genes found in enterococci.

\section{Conclusions}

In the last 8 years, microbiologists and clinicians have become aware of the existence of glycopeptide resistance in clinical isolates of gram-positive bacteria. Careful screening is necessary to ensure that these resistant organisms are detected. ${ }^{2}$ Although resistance to glycopeptides has been recognised in several bacterial genera, it is most relevant clinically when it appears in enterococci and staphylococci. In enterococci, several forms of resistance occur, each of which appears to result from production of novel peptidoglycan precursors with decreased affinity for glycopeptides. Staphylococci also have different forms of resistance, although the mechanisms differ from those in enterococci. The possibility of glycopeptide-resistant $S$. aureus remains a major concern, but there has been only one report of teicoplanin resistance and no reports of vancomycin resistance in clinical isolates of this species.

Mackey and colleagues ${ }^{22}$ recently stated that "Out- 
side VRLB (vancomycin-resistant lactic bacteria) and the rarely encountered Erysipelothrix rusiopathiae [sic], among the catalase-negative, gram-positive bacteria, only Enterococcus species may exhibit resistance to this antibiotic (vancomycin)". At this time such generalisations must be treated with caution and microbiologists should be wary of preconceptions of the vancomycin-sensitivity of other gram-positive species. Although glycopeptide resistance is recorded most often in the genera described above, it is unwise to assume that other gram-positive bacteria will always be sensitive. As an example, glycopeptide resistance was reported recently in Gemella haemolysans. ${ }^{23}$ Such

\section{References}

1. Johnson AP, Uttley AHC, Woodford N, George RC. Resistance to vancomycin and teicoplanin: an emerging clinical problem. Clin Microbiol Rev 1990; 3: 280-291.

2. Woodford N, Johnson AP, George RC. Detection of glycopeptide resistance in clinical isolates of gram-positive bacteria. J Antimicrob Chemother 1991; 28 : 483-486.

3. Woodford N, Johnson AP, George RC. Mechanisms and genetics of antimicrobial resistance in Enterococcus spp. PHLS Microbiol Digest 1992; 9: 151-154.

4. Dutka-Malen S, Molinas C, Arthur M, Courvalin P. The Vana glycopeptide resistant protein is related to D-alanyl-D-alanine ligase cell wall biosynthesis enzymes. Mol Gen Genet 1990; 224: 364-372.

5. Evers S, Sahm DF, Courvalin P. The vanB gene of vancomycin resistant Enterococcus faecalis V583 is structurally related to genes encoding D-Ala:D-Ala ligases and glycopeptide resistance proteins VanA and VanC. Gene 1993; 124: 143-144.

6. Dutka-Malen S, Molinas C, Arthur M, Courvalin P. Sequence of the vanC gene of Enterococcus gallinarum BM4174 encoding a D-alanine:D-alanine ligase-related protein necessary for vancomycin resistance. Gene 1992; 112: 53-58.

7. Messer J, Reynolds PE. Modified peptidoglycan precursors produced by glycopeptide-resistant enterococci. FEMS Microbiol Letts 1992; 94: 195-200.

8. Arthur M, Molinas C, Depardieu F, Courvalin P. Characterization of $\operatorname{Tn} 1546$, a $\operatorname{Tn} 3$-related transposon conferring glycopeptide resistance by synthesis of depsipeptide peptidoglycan precursors in Enterococcus faecium BM4147. J Bacteriol 1993; 175: 117-127.

9. Uttley AHC, George RC, Naidoo J et al. High-level vancomycin-resistant enterococci causing hospital infections. Epidemiol Infect 1989; 103: 173-181.

10. Fraimow H, Venuti E, Dean J. Mechanism of vancomycin dependence of a vancomycin-requiring clinical Enterococcus faecalis isolate. Program and Abstracts of the 33rd Interscience Conference on Antimicrobial Agents and Chemotherapy. 1993: 141: abstract 117.

11. Green M, Schlaes JH, Barbadora K, Schlaes DM. Vancomycin dependent Enterococcus faecium: a preliminary characterization. Program and Abstracts of the 33rd Interscience Conference on Antimicrobial Agents and Chemotherapy. 1993: 141 : abstract 118 .

12. Kaatz GW, Seo SM, Dorman NJ, Lerner SA. Emergence of resistance appears not to be an intrinsic property of this species, which suggests that this isolate had acquired resistance. If glycopeptides are considered to be appropriate therapy in a particular situation, any gram-positive isolates should be tested for sensitivity. Furthermore, it is advisable to test for resistance to both vancomycin and teicoplanin, as it is not always possible to infer cross-resistance or -sensitivity from tests with a single agent.

N. WoOdFord and A. P. JoHnson Antibiotic Reference Unit, Central Public Health Laboratory, 61 Colindale Avenue, London NW9 5HT.

teicoplanin resistance during therapy of Staphylococcus aureus endocarditis. $J$ Infect Dis 1990; 162: 103-108.

13. Biavasco F, Giovanetti E, Montanari MP, Lupidi R, Varaldo PE. Development of in-vitro resistance to glycopeptide antibiotics: assessment in staphylococci of different species. $J$ Antimicrob Chemother 1991; 27: 71-79.

14. Daum RS, Gupta S, Sabbagh R, Milewski WM. Characterization of Staphylococcus aureus isolates with decreased susceptibility to vancomycin and teicoplanin: isolation and purification of a constitutively produced protein associated with decreased susceptibility. $J$ Infect Dis 1992; 166: 1066-1072.

15. Sanyal D, Greenwood D. An electronmicroscope study of glycopeptide antibiotic-resistant strains of Staphylococcus epidermis. J Med Microbiol 1993; 39: 204-210.

16. Shlaes DM, Shlaes JH, Vincent S, Etter L, Fey PD, Goering RV. Teicoplanin-resistant Staphylococcus aureus expresses a novel membrane protein and increases expression of penicillin-binding protein 2 complex. Antimicrob Agents Chemother 1993; 37: 2432-2437.

17. O'Hare MD, Reynolds PE. Novel membrane proteins present in teicoplanin-resistant, vancomycin-sensitive, coagulasenegative Staphylococcus spp. J Antimicrob Chemother 1992; 30: 753-768.

18. Sanyal D, Johnson AP, George RC, Edwards R, Greenwood D. In vitro characteristics of glycopeptide resistant strains of Staphylococcus epidermidis isolated from patients on CAPD. J Antimicrob Chemother 1993; 32: 267-278.

19. Noble WC, Virani Z, Cree RGA. Co-transfer of vancomycin and other resistance genes from Enterococcus faecalis NCTC 12201 to Staphylococcus aureus. FEMS Microbiol Letts 1992; 93 : 195-198.

20. Huygens F. Vancomycin binding to cells walls of nonstreptococcal vancomycin-resistant bacteria. $J$ Antimicrob Chemother 1993; 32 : 551-558.

21. Bugg TDH, Wright GD, Dutka-Malen S, Arthur M, Courvalin $\mathrm{P}$, Walsh CT. Molecular basis for vancomycin resistance in Enterococcus faecium BM4147: biosynthesis of a depsipeptide peptidoglycan precursor by vancomycin resistance proteins VanH and VanA. Biochemistry 1991; 30: 10408-10415.

22. Mackey T, Lejeune V, Janssens M, Wauters G. Identification of vancomycin-resistant lactic bacteria isolated from humans. $J$ Clin Microbiol 1993; 31 : 2499-2501.

23. Reed C, Efstratiou A, Morrison D, Woodford N. Glycopeptide resistant Gemella haemolysans from blood. Lancet 1993; 342: 927-928. 